\title{
Space-Time Block Coded Spatial Modulation Based on Golden Code
}

\author{
Tien Dong Nguyen ${ }^{1}$, Xuan Nam Tran ${ }^{1}$, Vu Duc $\mathrm{Ngo}^{2}$, Minh Tuan $\mathrm{Le}^{2}$ \\ ${ }^{1}$ Le Quy Don Technical University, Ha Noi, Vietnam \\ ${ }^{2}$ Mobifone R\&D Center, Mobifone Corporation, Ha Noi, Vietnam \\ Correspondence: Tien Dong Nguyen, qttdong@gmail.com \\ Communication: received 14 April 2018, revised 2 August 2018, accepted 26 September 2018 \\ Online early access: 8 November 2018, Digital Object Identifier: 10.32913/rd-ict.vol2.no15.702 \\ The Area Editor coordinating the review of this article and deciding to accept it was Dr. Dau Son Hoang
}

\begin{abstract}
In this paper, a new Space-Time Block Coded Spatial Modulation (SM) scheme based on the Golden Code, called the Golden Coded Spatial Modulation (GC-SM), is proposed and analyzed. This scheme still keeps some main benefits of the Golden Code by satisfying the non-vanishing Space Time Block Code (STBC) criteria. In the signal constellation domain, the GC-SM spectral efficiency is twice that of the STBC-SM. In addition, simulation and theoretical results show that the GC-SM performance surpasses several SM schemes at the same spectral efficiency and antenna configuration. Furthermore, we study the impact of channel spatial correlation on the GC-SM performance. Finally, the GC-SM detection complexity is studied and compared with the existing SM schemes.
\end{abstract}

Keywords: Multiple-input multiple-output, space-time block code, golden code, spatial modulation.

\section{INTRODUCTION}

To overcome some Multi-Input Multi-Output (MIMO) system disadvantages, Mesleh et al. have recently proposed a Spatial Modulation (SM) scheme [1]. In the SM model, information bits are conveyed not only by conventional modulated symbols but also by the indices of transmit antennas to create a tridimensional (3-D) constellation. At a time slot, a modulated symbol is transmitted from one active antenna out of multiple transmit antennas. Therefore, the SM scheme totally avoids Inter-Channel Interference (ICI) at its receiver. Furthermore, the SM transmitters need only one radio frequency (RF) chain for transmission and require no synchronization among the transmit antennas. In [2], a Generalized 3-D Constellation design was proposed to enhance the SM system reliability by optimizing the symbol mapping method at each transmit anntena. Furthermore, based on a partial channel state information at the SM transmitter, a Bit-to-Symbol Mapping scheme was proposed in [3] to improve the SM performance. Although both mentioned schemes improve the SM performance, the SM spectral efficiency is still dependable on the number of transmit antennas, i.e., $\log _{2} n_{T}$ where $n_{T}$ is the number of transmit antennas. Therefore, Jintao et al. proposed the Generalized Spatial Modulation (GSM) scheme [4] which increases the SM spectral efficiency by simultaneously activating more than one transmit antenna. The number of additional bits carried by the antenna indices increases to $\left[\log _{2}\left(\begin{array}{l}n_{T} \\ n_{A}\end{array}\right)\right\rfloor$ where $n_{A}$ is the number of active antennas. However, since the GSM scheme requires multiple active transmit antennas to transmit symbols, its transmitter needs to utilize the equivalent number of radio frequency chains. In [5], the authors proposed a Quadrature Spatial Modulation (QSM) scheme utilizing two RF chains. In this model, by using the extra spatial dimension, the QSM spectral efficiency increases to $\log _{2}\left(n_{T}^{2}\right)+\log _{2} M$ bits per channel use (bpcu) where $M$ is the signal constellation order. Recently, based on multiple signal modulation techniques for two active antennas, an Enhanced Spatial Modulation (ESM) scheme have been proposed in [6] to improve the SM spectral efficiency. However, since the antenna indices are also an information source, these mentioned schemes' performances deteriorate under the spatial correlated environments.

Recently, various SM schemes have been proposed to overcome this drawback in the SM [7-9]. These schemes are robust under the spatial correlation effect by utilizing the Orthogonal Space Time Block Code (OSTBC) [10]. Basar et al. integrated the Alamouti Space Time Block Code (STBC) [11] in the SM to create the Space Time Block Coded Spatial Modulation (STBC-SM) scheme [7] which achieves the second order transmit diversity. This scheme not only improves the SM performance but also requires only a low-complexity detector at its receiver. The STBC-SM spectral efficiency is $\frac{1}{2} \log _{2} c+\log _{2} M$ (bpcu) 
where $c$ is the number of antenna combinations. Inspired by the result in [7], Xiaofeng et al. managed to improve the STBC-SM spectral efficiency by modifying the AlamoutiSTBC and cyclically shifting these matrices in two rows. The so-called High Rate Space Time Block Coded Spatial Modulation (STBC-CSM) [8] spectral efficiency becomes $\frac{1}{2} \log _{2} c+\log _{2} M$ (bpcu) where $c=\left\lfloor\left(n_{T}-1\right) n_{T}\right\rfloor_{2^{p}}$ is the total number of STBC-CSM codewords. In [9], by utilizing the Spatial Constellation (SC) matrices and the Alamouti matrix, Le et al. proposed the Spatially Modulated Orthogonal Space Time Block Coding (SM-OSTBC) scheme. The maximum SM-OSTBC spectral efficiency is $\left(n_{T}-2+\log _{2} M\right)$ (bpcu) when $n_{A}=n_{T}$. This scheme is only suitable for the MIMO systems that are equipped with more than three transmit antennas. However, in these mentioned schemes, transmitting the Alamouti-STBC reduces the spectral efficiency by half than that of the SM. To overcome this disadvantage, a new SM scheme, called the DSTTD-SM [12], is proposed to improve the SM spectral efficiency by applying the Double Space Time Transmit Diversity in the SM. However, compared with the STBCSM and the STBC-CSM, the DSTTD-SM utilizes all four transmit antennas with all antenna combinations to transmit symbols. In [13], a Spatially Modulated Space-Time Block Coding scheme, called DT-SM, is proposed by combining the SM with the Double Space Time Transmit Diversity (DSTTD) [14]. This scheme achieves higher spectral efficiency in the spatial domain than several schemes [7, 9]. However, the DT-SM transmiter implements at least four RF chains.

Inspired by the works in [12], we propose a new SpaceTime Block Coded Spatial Modulation (SM) scheme based on the Golden Code, called Golden Coded Spatial Modulation (GC-SM), for four transmit antennas. Compared with the DSTTD-SM, the GC-SM achieves the same spectral efficiency while utilizing only two RF chains. Furthermore, the GC-SM scheme enjoys the full benefits of the Golden code while improving SM spectral efficiency in the signal constellation domain. Simulation results show that the GCSM scheme outperforms several existing SM-based MIMO ones at the same spectral efficiency and same antenna configuration. Furthermore, the theoretical upper bound of the bit error probability (BEP) is derived to verify the GC-SM performance. Finally, the GC-SM complexity is calculated and compared with related SM schemes.

The rest of this paper is organized as follows. The proposed system model is presented in Section II. The SC codeword design and the signal detection algorithm are respectively introduced in Section III and Section IV. Performance evaluations are carried out in Section $\mathrm{V}$ and conclusions are drawn in Section VI.
Notations: $(\cdot)^{H}$ denotes the transpose of a matrix and $j^{2}=-1, \mathfrak{R}(\cdot)$ and $\mathfrak{J}(\cdot)$ are the real element and the imaginary element of a complex number, respectively, and $r^{*}$ is the conjugate number of $r$.

\section{The Proposed SM System Model}

The GC-SM scheme is considered in Figure 1. In this model, each block of $(l+4 m)$ data bits, coming to the GC$\mathrm{SM}$ transmitter, is separated in two parts. The first part with $l$ bits, is mapped into a $4 \times 2 \mathrm{SC}$ matrix, out of $K=2^{l} \mathrm{SC}$ matrices in the spatial constellation $\Omega_{S}$ while the remaining $4 m$ bits are modulated in $M$-QAM/ PSK modulators $(M=$ $\left.2^{m}\right)$. Then, these modulated symbols are arranged in the Golden Code structure [15] as follows:

$$
\mathbf{X}=\sqrt{\frac{1}{5}}\left[\begin{array}{ll}
a x_{1}+b x_{2} & a x_{3}+b x_{4} \\
c x_{3}+a x_{4} & d x_{1}+e x_{2}
\end{array}\right],
$$

where $a=(1+j \sigma(\theta)), b=(\theta-j), c=(1+j \sigma(\theta))$, $d=(1+j \theta), e=(\sigma(\theta)-j), \theta=\frac{1+\sqrt{5}}{2}, \sigma(\theta)=\frac{1-\sqrt{5}}{2}$ and $j^{2}=-1$. Finally, the $4 \times 2$ transmitted codeword $\mathbf{C}$ is formed as a product of $\mathbf{S}$ and $\mathbf{X}$, i.e., $\mathbf{C}=\mathbf{S X}$. The codeword $\mathbf{C}$ will be transmitted from four transmit antennas within two symbol periods.

The system equation is given by

$$
\mathbf{Y}=\mathbf{H C}+\mathbf{N}=\mathbf{H S X}+\mathbf{N},
$$

where $\mathbf{H}$ is an $n_{R} \times 4$ channel matrix and $\mathbf{N}$ is an $n_{R} \times 2$ noise matrix. All elements of the two matrices are assumed to be independently and identically distributed (i.i.d.) random variables with zero mean, unit variance, $\mathcal{N}(0,1)$, for the former and zero mean, $\sigma^{2}$ variance, $\mathcal{N}\left(0, \sigma^{2}\right)$, for the latter.

\section{SC CODEWORD DESIGN}

Based on the SC concept [9], a set of four SC codewords for four transmit antennas is proposed as follows

$$
\begin{array}{ll}
\mathbf{S}_{1}=\left[\begin{array}{cc}
1 & 0 \\
0 & 1 \\
0 & 0 \\
0 & 0
\end{array}\right] ; & \mathbf{S}_{2}=\left[\begin{array}{cc}
0 & 0 \\
0 & 0 \\
e^{j \theta} & 0 \\
0 & e^{-j \theta}
\end{array}\right] ; \\
\mathbf{S}_{3}=\left[\begin{array}{cc}
0 & 0 \\
e^{j 2 \theta} & 0 \\
0 & e^{-j 2 \theta} \\
0 & 0
\end{array}\right] ; \quad \mathbf{S}_{4}=\left[\begin{array}{cc}
e^{j 3 \theta} & 0 \\
0 & 0 \\
0 & 0 \\
0 & e^{-j 3 \theta}
\end{array}\right] .
\end{array}
$$

To keep the main benefits of the Golden Code, the optimal angle $\theta$ is exhaustively searched based on the non-vanishing determinant criterion [10] to find the maximum coding gain distance (CDG) as follows:

$$
\begin{aligned}
\delta_{\min } & =\underset{\mathbf{C} \neq \mathbf{C}^{\prime}}{\min \operatorname{det}}\left(\mathbf{C}-\mathbf{C}^{\prime}\right)^{H}\left(\mathbf{C}-\mathbf{C}^{\prime}\right), \\
\theta_{o} & =\underset{\theta}{\arg \max } \delta_{\min }(\theta) .
\end{aligned}
$$




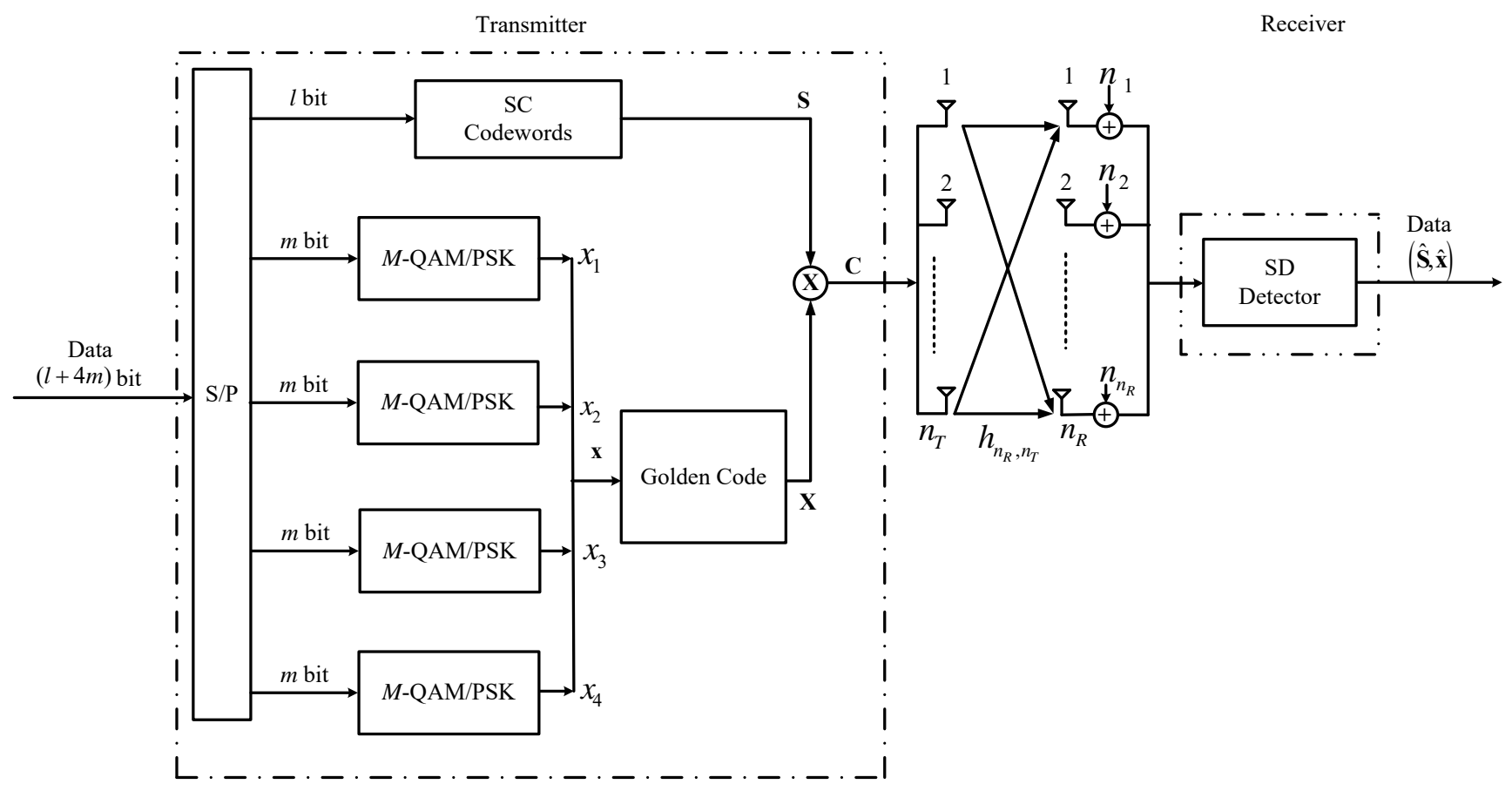

Figure 1. Block diagram of the GC-SM scheme.

TABLE I

OPTIMAL VALUES FOR $\theta$ AND THE CDG FOR DIFFERENT MODULATION TECHNIQUES

\begin{tabular}{|c|c|c|c|c|}
\hline Modulation & BPSK & 4QAM & 8QAM & 16QAM \\
\hline $\boldsymbol{\theta}$ & 0.72 & 1.26 & 0.46 & 0.26 \\
\hline$\delta_{\min }$ & 0.58 & 0.398 & 0.11 & 0.033 \\
\hline
\end{tabular}

The obtained angle and CDG results are summarized in Table I for different modulation techniques.

Since the number of SC codewords, $c$, is four, the achievable GC-SM spectral efficiency is given by

$$
C_{\mathrm{GC}-\mathrm{SM}}=\frac{1}{2} \log _{2} c+2 \log _{2} M(\mathrm{bpcu}),
$$

where $M$ is the modulation order. Compared with the STBC-SM spectral efficiency $\frac{1}{2} \log _{2} c+\log _{2} M$ (bpcu), the GC-SM spectral efficiency is twice higher than that of the STBC-SM with the same antenna configuration. Furthermore, the GC-SM has the same spectral efficiency with the DSTTD-SM while the GC-SM only utilizes two RF chains.

\section{GC-SM Signal DeteCtion}

\section{Signal Detection}

For a given matrix $\mathbf{S}_{k}, k=1,2, \ldots, K$, we are able to construct an equivalent $n_{R} \times 2$ matrix $\tilde{\mathbf{H}}_{k}=\mathbf{H S}_{k}$. Therefore, the system equation in (2) can be re-written as

$$
\mathbf{Y}=\tilde{\mathbf{H}}_{k} \mathbf{X}+\mathbf{N} .
$$

Arranging the $\mathbf{X}$ matrix structure into a column vector, we have

$$
\breve{\mathbf{x}}=\frac{1}{\sqrt{5}}\left[\begin{array}{c}
(1+j \sigma(\theta)) x_{1}+(\theta-j) x_{2} \\
(1-\theta) x_{3}+(1+j \sigma(\theta)) x_{4} \\
(1+j \sigma(\theta)) x_{3}+(\theta-j) x_{4} \\
(1+j \theta) x_{1}+(\sigma(\theta)-j) x_{2}
\end{array}\right] .
$$

After manipulating the $\breve{\mathbf{x}}$ vector in a real-valued form, this vector is given as

$$
\breve{\mathbf{x}}=\mathbf{G z},
$$

where $\mathbf{z}=\left[\begin{array}{llll}\mathfrak{R}\left(x_{1}\right) & \mathfrak{I}\left(x_{1}\right) & \cdots & \mathfrak{I}\left(x_{4}\right)\end{array}\right]^{T}$, and the generator matrix $\mathbf{G}$ is given as

$$
\mathbf{G}=\frac{1}{\sqrt{5}}\left[\begin{array}{cccccccc}
1 & -\sigma(\theta) & \theta & 1 & 0 & 0 & 0 & 0 \\
\sigma(\theta) & 1 & -1 & \theta & 0 & 0 & 0 & 0 \\
0 & 0 & 0 & 0 & -\theta & -1 & 1 & -\sigma(\theta) \\
0 & 0 & 0 & 0 & 1 & -\theta & \sigma(\theta) & 1 \\
0 & 0 & 0 & 0 & 1 & -\sigma(\theta) & \theta & 1 \\
0 & 0 & 0 & 0 & \sigma(\theta) & 1 & -1 & \theta \\
1 & -\theta & \sigma(\theta) & 1 & 0 & 0 & 0 & 0 \\
\theta & 1 & -1 & \sigma(\theta) & 0 & 0 & 0 & 0
\end{array}\right] .
$$

Then, Equation (9) is rewritten as

$$
\mathbf{v}=\overline{\mathbf{H}}_{k} \mathbf{G z}+\mathbf{w}=\mathbf{M}_{k} \mathbf{z}+\mathbf{w},
$$

where

$$
\overline{\mathbf{H}}_{k}=\left[\begin{array}{cc}
\mathbf{U} & \mathbf{0}_{2 n_{R} \times 4} \\
\mathbf{0}_{2 n_{R} \times 4} & \mathbf{U}
\end{array}\right],
$$




$$
\mathbf{U}=\left[\begin{array}{cccc}
\mathfrak{R}\left(\tilde{h}_{11}\right) & -\mathfrak{I}\left(\tilde{h}_{11}\right) & \cdots & -\mathfrak{I}\left(\tilde{h}_{12}\right) \\
\mathfrak{I}\left(\tilde{h}_{11}\right) & \mathfrak{R}\left(\tilde{h}_{11}\right) & \cdots & \mathfrak{R}\left(\tilde{h}_{12}\right) \\
\vdots & \vdots & \ddots & \vdots \\
\mathfrak{R}\left(\tilde{h}_{n_{R} 1}\right) & -\mathfrak{J}\left(\tilde{h}_{n_{R} 1}\right) & \cdots & -\mathfrak{J}\left(\tilde{h}_{n_{R}}\right) \\
\mathfrak{I}\left(\tilde{h}_{n_{R} 1}\right) & \mathfrak{R}\left(\tilde{h}_{n_{R} 1}\right) & \cdots & \mathfrak{R}\left(\tilde{h}_{n_{R} 2}\right)
\end{array}\right],
$$

$\mathbf{v}=\left[\begin{array}{llll}\mathfrak{R}\left(y_{11}\right) & \mathfrak{J}\left(y_{11}\right) & \cdots & \mathfrak{J}\left(y_{n_{R} 2}\right)\end{array}\right]^{T}$, and $\mathbf{w}$ has the same structure as $\mathbf{v}$.

Equation (11) is now similar to the system equation of a conventional spatial multiplexing scheme. Therefore, the Sphere Decoders (SD) in $[16,17]$ can be used to detect $\mathbf{z}$ for a given $\mathbf{S}_{k}$ as follows:

$$
(\hat{\mathbf{z}})_{k}=\underset{\mathbf{z} \in \Omega_{N}}{\arg \min }\left\|\mathbf{t}_{k}-\mathbf{R}_{k} \mathbf{z}\right\|^{2},
$$

where $\mathbf{t}_{k}=\mathbf{Q}_{k}^{H} \mathbf{v}, \mathbf{M}_{k}=\mathbf{Q}_{k} \mathbf{R}_{k}$, and $\Omega_{N}$ is the set of integers corresponding to $M$-QAM constellation.

After that, the index $k$ of the transmitted SC codeword is determined as follows:

$$
\hat{k}=\underset{k=1, \ldots, K}{\arg \min }\left\|\mathbf{t}_{k}-\mathbf{R}_{k}(\mathbf{z})_{k}\right\|^{2}-\mathbf{t}_{k}^{H} \mathbf{t}_{k} .
$$

Finally, the information bits are recovered from the detected SC codeword and the detected signal vector $\left(\hat{\mathbf{S}}_{k}, \hat{\mathbf{x}}_{k}\right)$ at the GC-SM receiver.

\section{Complexity Analysis}

It is assumed that each real math operation such as a real addition or a real multiplication is considered as a floating point operation (flop). As a result, a complex multiplication requires six flops while a complex addition requires two flops. The GC-SM complexity is calculated and compared with related SM-based MIMO schemes with the same structure. All schemes apply suitable modulation techniques to obtain the same spectral efficiency and use the sphere decoder at their receivers. Furthermore, the channel is assumed to remain unchanged within $T$ symbol periods.

In the pre-processing state, the complexity of computing $\tilde{\mathbf{H}}_{k}$ in (7), $\mathbf{M}_{k}$ in (11), and QR decomposition of $\mathbf{M}_{k}$ in (12) is given as

$$
\rho_{\text {pre }}=\frac{2}{T}\left(1032 n_{R}+4 n_{R} n_{T}+36\right) K+\left(64 n_{R}+7\right) K .
$$

Therefore, the GC-SM complexity is calculated as

$$
\rho_{\mathrm{GC}-\mathrm{SM}}=\frac{\rho_{\mathrm{pre}}+\rho_{s}}{4 m+2},
$$

where $\rho_{s}$ is the average number of operations within SD searching stage.

Figure 2 compares the detection complexity of the GCSM with the related SM schemes such as the STBC-SM [7], the STBC-CSM [8], the SM-DC [18], and the ESM [6], equipped with four transmit and four receive, i.e. $(4,4)$,

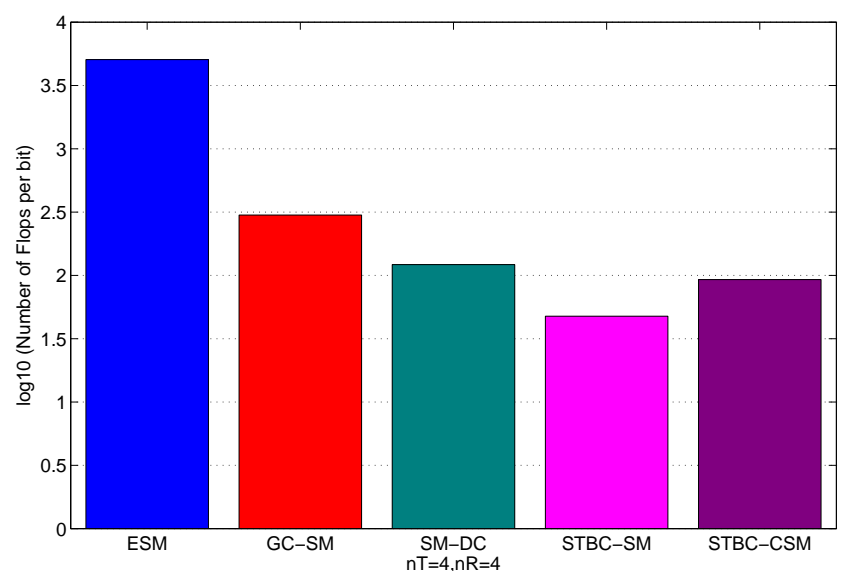

Figure 2. Complexity comparison between GC-SM, STBC-SM, STBCCSM, SM-DC, and ESM at the spectral efficiency of 5 bpcu, SNR of $10 \mathrm{~dB}$, with four transmit antennas, four receive antennas, and $T=60$ symbol periods.

antennas at the spectral efficiency of $5 \mathrm{bpcu}$. Figure 2 shows that the complexity of the GC-SM is higher than those of the STBC-SM, the SM-DC, and the STBC-CSM, but lower than that of the ESM. This is due to the fact that the Golden Code structure in the GC-SM is more complex than that of the Alamouti STBC in the STBC-SM and the STBCCSM. The ESM has the highest complexity because it needs to use the maximum likelihood detector to estimate the transmitted symbols using multiple signal constellations. However, as will be shown in Section V, the proposed GCSM outperforms all others in terms of BER performance.

\section{Theoretical Upper Bound for the BEP of the GC-SM}

The bit error probability (BEP) of the GC-SM can be derived from the pairwise error probability (PEP) $P\left(\mathbf{C}_{i} \rightarrow\right.$ $\mathbf{C}_{j}$ ) that the transmitted codeword matrix $\mathbf{C}_{i}$ is mistakenly decoded for matrix $\mathbf{C}_{j}[19]$ as follows:

$$
P_{b} \leq \frac{1}{N} \sum_{i=1}^{N} \sum_{j=1}^{N} \frac{P\left(\mathbf{C}_{i} \rightarrow \mathbf{C}_{j}\right) w_{i, j}}{\log _{2} N},
$$

where $N=K M^{4}$ and $w_{i, j}$ is the number of erroneous bits between the matrices $\mathbf{C}_{i}$ and $\mathbf{C}_{j}$.

The conditional PEP of the GC-SM system is calculated as

$$
P\left(\mathbf{C}_{i} \rightarrow \mathbf{C}_{j} \mid \mathbf{H}\right)=Q\left(\sqrt{\frac{\gamma}{2}} d^{2}\left(\mathbf{C}_{i}, \mathbf{C}_{j}\right)\right),
$$

where $Q(x)=(1 / 2 \pi) \int_{x}^{\infty} e^{-y^{2} / 2} d y$. 
From [20], the PEP is given as

$$
P\left(\mathbf{C}_{i} \rightarrow \mathbf{C}_{j}\right)=\frac{1}{\pi} \int_{0}^{\frac{\pi}{2}}\left(\frac{1}{1+\frac{\gamma \lambda_{i, j, 1}}{4 \sin ^{2} \phi}}\right)^{n_{R}}\left(\frac{1}{1+\frac{\gamma \lambda_{i, j, 2}}{4 \sin ^{2} \phi}}\right)^{n_{R}} d \phi,
$$

where $\lambda_{i, j, 1}$ and $\lambda_{i, j, 2}$ are the eigenvalues of the distance matrix $\left(\mathbf{C}_{i}-\mathbf{C}_{j}\right)\left(\mathbf{C}_{i}-\mathbf{C}_{j}\right)^{H}$.

Converting the (18), we have

$$
\begin{aligned}
P\left(\mathbf{C}_{i} \rightarrow \mathbf{C}_{j}\right) & =\frac{1}{\pi} \int_{0}^{\frac{\pi}{2}}\left(\frac{\sin ^{2} \phi}{\sin ^{2} \phi+\frac{\gamma \lambda_{i, j, 1}}{4}}\right)^{n_{R}}\left(\frac{\sin ^{2} \phi}{\sin ^{2} \phi+\frac{\gamma \lambda_{i, j, 2}}{4}}\right)^{n_{R}} d \phi \\
& =\frac{1}{\pi} \int_{0}^{\frac{\pi}{2}}\left(\frac{\sin ^{2} \phi}{\sin ^{2} \phi+c_{1}}\right)^{m}\left(\frac{\sin ^{2} \phi}{\sin ^{2} \phi+c_{2}}\right)^{m} d \phi,
\end{aligned}
$$

where $c_{1}=\frac{\gamma \lambda_{i, j, 1}}{4}, c_{2}=\frac{\gamma \lambda_{i, j, 2}}{4}$, and $n_{R}=m$.

From [20], the closed form of the (19) is presented by

$$
\begin{array}{r}
P\left(\mathbf{C}_{i} \rightarrow \mathbf{C}_{j}\right)=\frac{\left(c_{1} / c_{2}\right)^{m-1}}{2\left(1-c_{1} / c_{2}\right)^{2 m-1}}\left[\sum_{k=0}^{m-1}\left(\frac{c_{2}}{c_{1}}-1\right)^{k} B_{k} I_{k}\left(c_{2}\right)\right. \\
\left.-\frac{c_{1}}{c_{2}} \sum_{k=0}^{m-1}\left(1-\frac{c_{1}}{c_{2}}\right)^{k} C_{k} I_{k}\left(c_{1}\right)\right]
\end{array}
$$

where

$$
\begin{aligned}
& B_{k} \triangleq \frac{A_{k}}{\left(\begin{array}{c}
2 m-1 \\
k
\end{array}\right)}, \\
& C_{k} \triangleq \sum_{n=0}^{m-1} \frac{\left(\begin{array}{c}
k \\
n
\end{array}\right)}{\left(\begin{array}{c}
2 m-1 \\
n
\end{array}\right)} A_{n}, \\
& A_{k} \triangleq(-1)^{m-1+k} \frac{\left(\begin{array}{c}
m-1 \\
k
\end{array}\right)}{(m-1) !} \prod_{\substack{n=1 \\
n \neq k+1}}^{m}(2 m-n),
\end{aligned}
$$

and

$$
I_{k}(c)=1-\sqrt{\frac{c}{c+1}}\left[1+\sum_{n=1}^{k} \frac{(2 n-1) ! !}{n ! 2^{n}(1+c)^{n}}\right],
$$

where the double factorial notation denotes the product of only odd integers from 1 to $2 k-1$.

\section{Simulation Results}

In this section, the GC-SM performance is evaluated and compared with several related SM systems such as the ESM, the STBC-SM, the SM-DC, and the STBCCSM using different modulation techniques. The number of transmit antennas, receive antennas, and active antennas in each scheme are represented respectively by $\left(n_{T}, n_{R}, n_{A}\right)$. Furthermore, it is assumed that all schemes employ the sphere detector at the receiver while the ESM uses the ML detector.

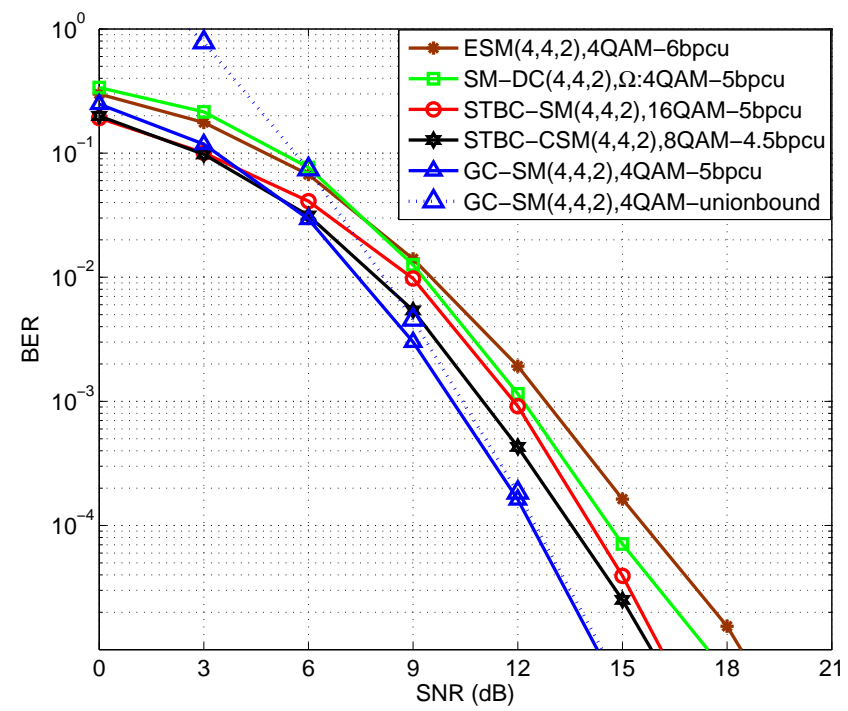

Figure 3. Performance comparison between GC-SM, STBC-SM, SM-DC, ESM, and STBC-CSM with $(4,4)$ antennas and the spectral efficiency of 5 bpcu.

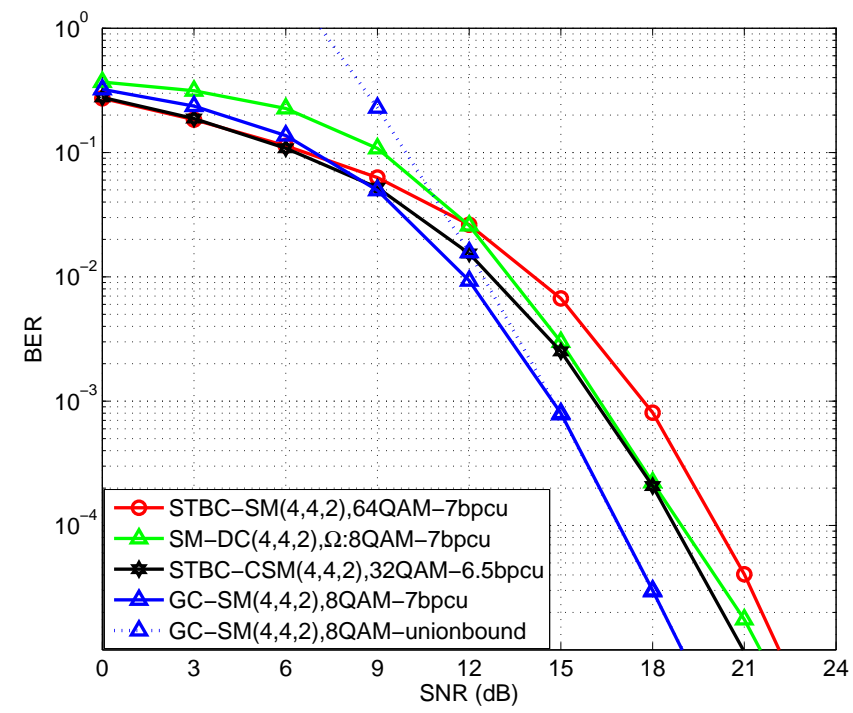

Figure 4. Performance comparison between GC-SM, STBC-SM, SM-DC, and STBC-CSM with $(4,4)$ antennas and the spectral efficiency of $7 \mathrm{bpcu}$.

In Figure 3, the GC-SM performance is compared with that of the ESM, the SM-DC, the STBC-SM, and the STBC-CSM at the spectral efficiency of $5 \mathrm{bpcu}$. The GC$\mathrm{SM}$ was shown to outperform the related schemes at the high SNR region. Particularly, at BER $=10^{-3}$, the GCSM yielded SNR gains of $0.9 \mathrm{~dB}, 2 \mathrm{~dB}, 2.1 \mathrm{~dB}$, and $2.7 \mathrm{~dB}$ over the STBC-CSM, the STBC-SM, the SM-DC, and the ESM, respectively. However, the achievable spectral efficiency of the ESM was 6 bpcu compared with 5 bpcu of the GC-SM with the same antenna configuration and same signal modulation. In Figure 4, the GC-SM performance is compared with that of the STBC-SM, the SM-DC, and 
the STBC-CSM at the spectral efficiency of $7 \mathrm{bpcu}$. The GC-SM was shown to outperform the related schemes at the high SNR region. Particularly, at BER $=10^{-3}$, the GC$\mathrm{SM}$ yielded SNR gains of $1.5 \mathrm{~dB}, 1.6 \mathrm{~dB}$, and $3 \mathrm{~dB}$ over the STBC-CSM, the SM-DC, and the STBC-SM, respectively. Therefore, compared with these schemes, the GC-SM scheme can save transmit power. It shows in Figure 3 and 4 that the theoretical results coincide with the simulation results at the high SNR region.

\section{The GC-SM under Spatial Correlation Effect}

In order to show the effectiveness of the proposed GC$\mathrm{SM}$, we evaluated its performance under a more realistic spatially correlated channel. From [21], the modified MIMO channel matrix under the spatial correlation effect at both transmitter and receiver is given by

$$
\overline{\mathbf{H}}=\mathbf{R}_{R}^{1 / 2} \mathbf{H} \mathbf{R}_{T}^{1 / 2},
$$

where $\mathbf{R}_{T}$ and $\mathbf{R}_{R}$ are an $\left(n_{T} \times n_{T}\right)$ transmit spatial correlation matrix and an $\left(n_{R} \times n_{R}\right)$ receive spatial correlation matrix, respectively. Each element of these matrices is derived from the exponential correlation matrix model [22]: $r_{i j}=r_{j i}^{*}$ for $i<j$ or $r=r^{j-i}$ for $i \geq j$ where $r$ is the correlation coefficient of the neighboring transmit and receive antennas.

Figure 5 illustrates performances of the GC-SM, the STBC-SM, the ESM, the SM-DC, and the STBC-CSM, equipped with four transmit and four receive antennas at a suitable spectral efficiency of $5 \mathrm{bpcu}$ and the medium correlation factor $r=0.5$. The performance of the GC-SM

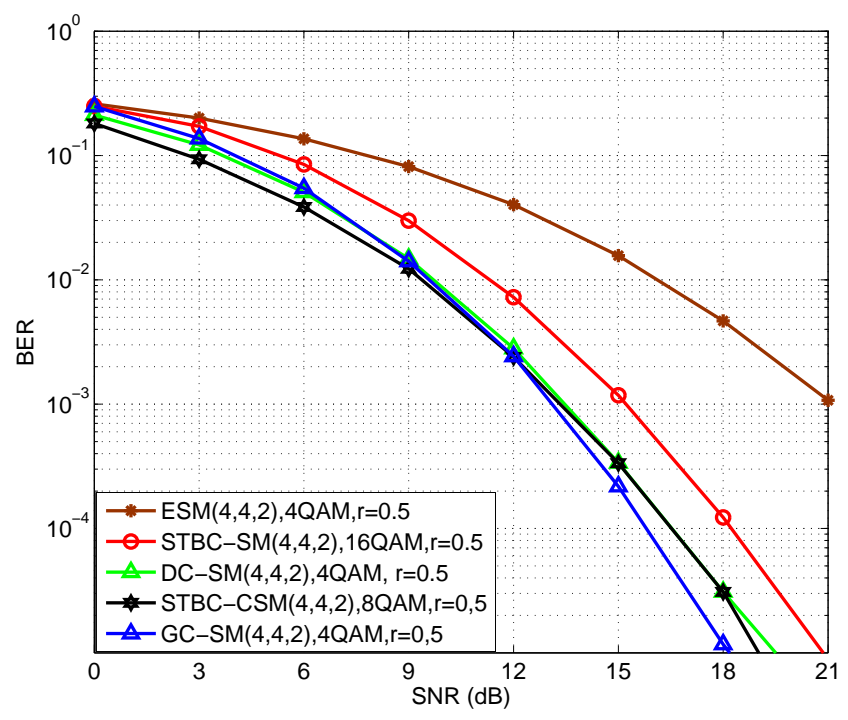

Figure 5. Performance comparison between the GC-SM, the STBCSM, the SM-DC, the ESM, and the STBC-CSM with $(4,4)$ antennas, the spectral efficiency of $5 \mathrm{bpcu}$, and $r=0.5$. was slightly affected by the spatial correlation, however, it was still more robust than the others, especially at the high SNR region. At BER $=10^{-3}$, the GC-SM offered about $0.2 \mathrm{~dB}, 0.3 \mathrm{~dB}, 1.8 \mathrm{~dB}$, and $6 \mathrm{~dB}$ SNR gains over the STBC-CSM, the SM-DC, the STBC-SM, and the ESM, respectively. At SNR $<10 \mathrm{~dB}$, the STBC-CSM achieves the best performance. It can be explained that the transmit STBC-CSM matrices, which maximize the coding gains, are optimally chosen from a set of STBC-CSM matrices. The ESM has the worst performance. This result can be explained that as the Euclidean distances between the antenna indices under the correlation effect get smaller, the BER of this scheme increases.

\section{Conclusions And Future Works}

In this paper, we have proposed a new SM-based MIMO system with four transmit antennas, called GC-SM, by embedding the Golden Code in the SM. The GC-SM achieves higher spectral efficiency than that of the STBC-SM and the STBC-CSM with the same antenna configuration. The proposed GC-SM was shown to outperform several related SM schemes at the same spectral efficiency with suitable cost of detection complexity. Besides, the GC-SM PEP union bound is derived to verify the simulation results. Furthermore, the GC-SM is shown to be robust under spatially correlated fading channels.

From results presented in the paper, as the proposed scheme works for MIMO scenario equipped with four transmit and four receive antennas, in the near future we will focus on designing a general procedure for SC codewords operating with an arbitrary number of transmit antennas. We also look for efficient low-complexity detection algorithms for MIMO-SM schemes. Besides, the bit error probability upper bound of MIMO-SM schemes will be investigated at the low SNR region.

\section{ACKNOWLEDGMent}

This work is sponsored by National Foundation for Science and Technology Development (Nafosted) under project number 102.02-2015.23.

\section{REFERENCES}

[1] R. Mesleh, H. Haas, S. Sinanovic, C. W. Ahn, and S. Yun, "Spatial modulation," IEEE Transactions on Vehicular Technology, vol. 57, no. 4, pp. 2228-2241, 2008.

[2] S. Guo, H. Zhang, P. Zhang, D. Wu, and D. Yuan, "Generalized 3-d constellation design for spatial modulation," IEEE Transactions on Communications, vol. 65, no. 8, pp. 33163327, 2017.

[3] S. Althunibat and R. Mesleh, "A bit-to-symbol mapping scheme for spatial modulation with partial channel state information," IEEE Communications Letters, vol. 21, no. 5, pp. 995-998, 2017. 
[4] J. Wang, S. Jia, and J. Song, "Generalised spatial modulation system with multiple active transmit antennas and low complexity detection scheme," IEEE Transactions on Wireless Communications, vol. 11, no. 4, pp. 1605-1615, 2012.

[5] R. Mesleh, S. S. Ikki, and H. M. Aggoune, "Quadrature spatial modulation," IEEE Transactions on Vehicular Technology, vol. 64, no. 6, pp. 2738-2742, 2015.

[6] C.-C. Cheng, H. Sari, S. Sezginer, and Y. T. Su, "Enhanced spatial modulation with multiple signal constellations," IEEE Transactions on Communications, vol. 63, no. 6, pp. 22372248, 2015.

[7] E. Basar, U. Aygolu, E. Panayirci, and H. V. Poor, "Spacetime block coded spatial modulation," IEEE Transactions on Communications, vol. 59, no. 3, pp. 823-832, 2011.

[8] X. Li and L. Wang, "High rate space-time block coded spatial modulation with cyclic structure," IEEE Communications Letters, vol. 18, no. 4, pp. 532-535, 2014.

[9] M.-T. Le, V.-D. Ngo, H.-A. Mai, X. N. Tran, and M. Di Renzo, "Spatially modulated orthogonal space-time block codes with non-vanishing determinants," IEEE Transactions on Communications, vol. 62, no. 1, pp. 85-99, 2014.

[10] V. Tarokh, H. Jafarkhani, and A. R. Calderbank, "Space-time block codes from orthogonal designs," IEEE Transactions on Information theory, vol. 45, no. 5, pp. 1456-1467, 1999.

[11] S. M. Alamouti, "A simple transmit diversity technique for wireless communications," IEEE Journal on selected areas in communications, vol. 16, no. 8, pp. 1451-1458, 1998.

[12] M. C. Park, B. G. Jo, and D. S. Han, "Double spacetime transmit diversity with spatial modulation," Electronics Letters, vol. 51, no. 25, pp. 2155-2156, 2015.

[13] M.-T. Le, T.-D. Nguyen, X.-N. Tran, and V.-D. Ngo, "On the combination of double space time transmit diversity with spatial modulation," IEEE Transactions on Wireless Communications, vol. 17, no. 1, pp. 170-181, 2018.

[14] Texas Instruments, "Double-sttd scheme for hsdpa systems with four transmit antennas: Link level simulation results," TSG-R WG1 document, TSGRI\#20(01)0458, 2001.

[15] J.-C. Belfiore, G. Rekaya, and E. Viterbo, "The golden code: a $2 \times 2$ full-rate space-time code with nonvanishing determinants," IEEE Transactions on information theory, vol. 51, no. 4, pp. 1432-1436, 2005.

[16] E. Viterbo and E. Biglieri, "A universal decoding algorithm for lattice codes," in Proceedings of the GRETSI, France, 1993, pp. 611-614.

[17] M. O. Damen, H. El Gamal, and G. Caire, "On maximumlikelihood detection and the search for the closest lattice point," IEEE Transactions on information theory, vol. 49, no. 10 , pp. 2389-2402, 2003.

[18] L. Wang and Z. Chen, "Spatially modulated diagonal space time codes," IEEE Communications Letters, vol. 19, no. 7, pp. 1245-1248, 2015.

[19] V. Tarokh, N. Seshadri, and A. R. Calderbank, "Space-time codes for high data rate wireless communication: Performance criterion and code construction," IEEE transactions on information theory, vol. 44, no. 2, pp. 744-765, 1998.

[20] M. K. Simon and M.-S. Alouini, Digital communication over fading channels. John Wiley \& Sons, 2005, vol. 95.

[21] A. Paulraj, R. Nabar, and D. Gore, Introduction to spacetime wireless communications. Cambridge university press, 2003.

[22] S. L. Loyka, "Channel capacity of mimo architecture using the exponential correlation matrix," IEEE Communications letters, vol. 5, no. 9, pp. 369-371, 2001.

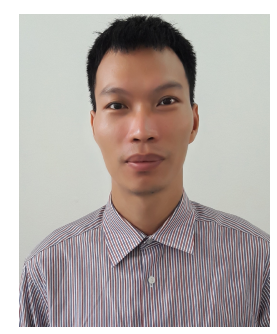

Tien Dong Nguyen was born in Quang Tri, Vietnam in 1982. He received his B.E. degree and M.S. degree both in electronic engineering from Le Quy Don Technical University,Vietnam, in 2006 and 2012, respectively. From 2006 to 2010 he worked as a lecturer at Telecommunication University, Vietnam. He is currently working toward his Ph.D. degree in electronic engineering at Le Quy Don Technical University.

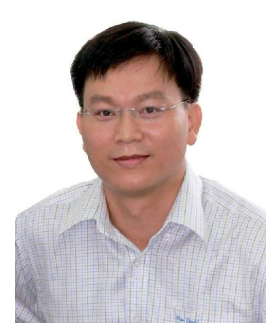

Xuan Nam Tran is currently an associate professor and head of the research group in advanced wireless communications in Le Quy Don Technical University Vietnam. He received his Master of Engineering (ME) in Telecommunications Engineering from University of Technology Sydney, Australia in 1998, and Doctor of Engineering in Electronic Engineering from The University of Electro-Communications, Japan in 2003. From November 2003 to March 2006 he was a research associate at the Information and Communication Systems Group, Department of Information and Communication Engineering, The University of Electro- Communications, Tokyo, Japan. Dr. Tran research interests are in the areas of space-time signal processing for communications such as adaptive antennas, space-time coding, MIMO, spatial modulation and cooperative communications. Dr. Tran is a recipient of the 2003 IEEE AP-S Japan Chapter Young Engineer Award, and a co-recipient of two best papers from The 2012 International Conference on Advanced Technologies for Communications and The 2014 National Conference on Electronics, Communications and Information Technology. He is a member of IEEE, IEICE and the Radio- Electronics Association of Vietnam (REV). 


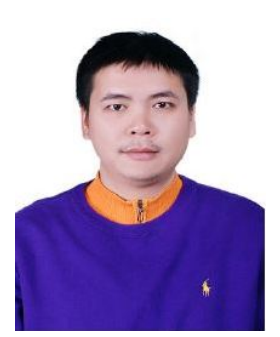

Vu Duc Ngo is currently a lecturer at School of Electronics and Telecommunications, Hanoi University of Science and Technology, and a researcher at MobiFone Research and development Center, MobiFone corporation, Vietnam. He received the Ph.D. degree from Korea Advanced Institute of Science and Technology in 2011. During 2007-2009 he was a Co-founder and CTO of Wichip Technologies Inc, USA. Since 2009, he is also a Co-founder and Director of uVision Jsc, Vietnam. Since November 2012 Dr. Ngo has been serving as a BoM member of the National Program on Research, Training, and Construction of High-Tech Engineering Infrastructure of Vietnam. His research interests are in the fields of SoC, NoC design and verification, and VLSI design for multimedia codecs as well as wireless communications PHY layer. Dr. Ngo is recipient of IEEE 2006 ICCES and IEEE 2012 ATC best paper awards. Dr. Ngo is a member of IEEE.

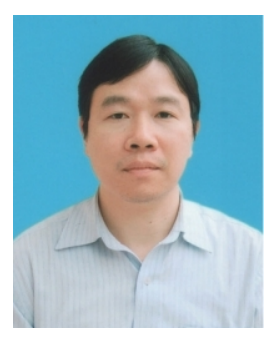

Minh Tuan Le was born in Thanh Hoa, Vietnam, in 1976. He received his B.E. degree in electronic engineering from Hanoi University of Science and Technology, Vietnam in 1999, M.S. degree and Ph.D. degree both in electrical engineering from Information and Communication University, which is currently the Department of Electrical and Engineering of Korean Advanced Institute of Science and Technology (KAIST), Daejon, Korea, in 2003 and 2007, respectively. From 1999 to 2001 and from 2007 to 2008 he worked as a lecturer at Posts and Telecommunication Institute of Technology (PTIT), Vietnam. From November 2012 to 2015, he worked at Hanoi Department of Science and Technology, Vietnam. $\mathrm{He}$ is currently working at MobiFone Reasearch and Development Center, MobiFone Corporation, Vietnam. His research interests include space-time coding, space-time processing, and MIMO systems. Dr. Le is the recipient of the 2012 ATC Best Paper Award from the Radio Electronics Association of Vietnam (REV) and the IEEE Communications Society. He is a member of IEEE. 